\title{
Cirugia laparoscópica en el tratamiento de enfermedades adrenales: experiencia en 200 casos
}

\author{
Castillo $\mathrm{O}^{1,2}$, Cortés $\mathrm{O}^{1}$, Kerkebe $\mathrm{M}^{3}$, Pinto $\mathrm{I}^{1}$, Arellano $\mathrm{L}^{4}$, Contreras $\mathrm{M}^{5}$. \\ ${ }^{1}$ Sección de Endourología y Laparoscopía Urológica. Clínica Santa María. ${ }^{2}$ Departamento de Urología. Facultad \\ de Medicina. Universidad de Chile. ${ }^{3}$ Servicio de Urología. Hospital Dipreca y \\ Servicio de Urología. Hospital San Juan de Dios. ${ }^{4}$ Departamento de Anatomía Patológica. Facultad de \\ Medicina. Universidad de Chile. ${ }^{5}$ Servicio de Anestesiología. Clínica Santa María. Chile.
}

Actas Urol Esp. 2006;30(9):926-932

\section{RESUMEN}

CIRUGÍA LAPAROSCÓPICA EN EL TRATAMIENTO DE ENFERMEDADES ADRENALES: EXPERIENCIA EN 200 CASOS.

Objetivo: Presentamos nuestra experiencia en adrenalectomía laparoscópica, luego de 10 años de adoptar la técnica laparoscópica como primera opción en la cirugía suprarrenal.

Métodos: Se incluyeron 200 cirugías adrenales laparoscópicas realizadas en forma consecutiva en 183 pacientes con patología quirúrgica suprarrenal entre noviembre de 1994 y noviembre de 2005 . Fueron 67 (36,6\%) hombres y 116 (63,4\%) mujeres con una edad promedio de 49,1 años (rango: 8 meses - 78 años).

Resultados: Los diagnósticos clínicos más frecuentes fueron hiperaldosteronismo $(17,5 \%)$, cáncer metastásico $(15,8 \%)$, feocromocitoma $(15,3 \%)$, sindrome de Cushing $(7,1 \%)$, quiste adrenal $(4,9 \%)$ y mielolipoma $(2,7 \%)$. Se realizaron 164 adrenalectomías totales, 29 adrenalectomías parciales y 7 marsupializaciones de quistes adrenales. El tiempo operatorio promedio fue de 82,6 minutos (rango: 25-240 minutos) y el tiempo de hospitalización fue de 2,5 días (rango: 110 días). El tamaño de la glándula y/o tumor suprarrenal varió entre 1 y $14 \mathrm{~cm}$ (promedio: 5,6 cm). La tasa de complicaciones fue del 6\%. En 8 pacientes además de la cirugía adrenal, se realizó otro procedimiento quirúrgico laparoscópico: colecistectomía (2), marsupialización de quiste renal (2), nefrectomía en bloque (2), nefrectomía parcial por tumor (1) y quistectomía pancreática (1). A un paciente a quien se le realizó adrenalectomía laparoscópica derecha, se le practicó nefrolitotomía percutánea ipsilateral en el mismo acto quirúrgico.

Conclusión: La experiencia acumulada en 200 cirugias adrenales laparoscópicas nos ha permitido manejar satisfactoriamente patologias endocrinas como aldosteroma, feocromocitoma, síndrome de Cushing, y entidades raras como quistes y mielolipomas; además, extender los beneficios de un procedimiento mínimamente invasivo para grandes masas adrenales y casos oncológicos seleccionados.

Palabras clave: Adrenalectomía. Glándula suprarrenal. Laparoscopía.

\section{ABSTRACT}

LAPAROSCOPIC SURGERY IN THE TREATMENT OF ADRENAL PATHOLOGY: EXPERIENCE WITH 200 CASES.

Objective: We are presenting our experience in laparoscopic adrenalectomy, after adopting the laparoscopic technique for 10 years as a primary option for suprarenal surgery.

Methods: We included 200 laparoscopic adrenal surgeries performed in a consecutive manner in 183 patients with surgical suprarenal pathology between November 1994 and November 2005. Sixty-seven (36.6\%) patients were male and 116 (63.4\%) were female, with an average age of 49.1 years (age range 8 months to 78 years).

Results: The most frequents clinical diagnosis were hyperaldosteronism (17.5\%), metastatic cancer (15.8\%), pheochromocytoma (15.3\%), Cushing syndrome (7.1\%), adrenal cyst (4.9\%) and myelolipoma (2.7\%). A total of 164 total adrenalectomies, 29 partial adrenalectomies and 7 marsupializations of adrenal cysts were performed. Mean surgical time was 82.6 minutes (range 25 to 240 minutes) and mean hospitalization time was 2.5 days (range 1-10 days). The size of the suprarenal gland and/or tumor varied between 1 and $14 \mathrm{~cm}$ (average $5.6 \mathrm{~cm}$ ). The rate of complication was $6 \%$. In 8 of the patients, there was another laparoscopic procedure besides the adrenal surgery: cholecystectomies (2), marsupialization of a renal cyst (2), block nephrectomy (2), partial nephrectomy for a tumor (1) and pancreatic cystectomy (1). One patient underwent a right laparoscopic adrenalectomy and an ipsilateral percutaneous nephrolithotomy in the same surgical act.

Conclusion: The accumulated experience with 200 laparoscopic adrenal procedures has allowed the management of endocrine pathologies, such as, aldosteroma, pheochromocytoma, Cushing syndrome and rare entities, such as, cysts, myelolipomas in a suitable manner. Additionally, it has permitted us to extend the benefits of a minimally invasive procedure for large adrenal masses and selected oncology cases.

Keywords: Adrenalectomy. Adrenal glands. Laparoscopy. 
$\mathrm{S}_{\mathrm{s}}^{\mathrm{oo}}$ n variadas las patologías derivadas de la glándula suprarrenal, y en la mayoría de los casos, es el clínico quien orienta su estudio y manejo. Sin embargo, hay entidades que demandan tratamiento quirúrgico haciendo que el enfoque del paciente sea interdisciplinario: es el caso del aldosteroma, feocromocitoma y la enfermedad de Cushing. En ocasiones, como hallazgo incidental o por sospecha clínica, en estudios de imágenes se encuentran adenomas no funcionantes, o entidades raras como quistes o mielolipomas, los cuales requieren de riguroso criterio clínico para definir su manejo.

Antes de la década de los años 90 el abordaje quirúrgico de la glándula suprarrenal exigía una gran incisión abdominal y se asociaba a una alta tasa de morbilidad. Esta gran incisión se hacía necesaria para exponer un órgano pequeño, friable, con abundante y delicada vasculatura, de localización retroperitoneal ${ }^{1}$.

A pesar de los avances técnicos en cirugía abierta, en el manejo médico y anestésico de los pacientes sometidos a cirugía suprarrenal, el cambio sólo sucedió cuando Gagner $^{2}$, en 1992, exportó su experiencia inicial de adrenalectomía laparoscópica transperitoneal en tres pacientes con patología adrenal benigna: sindrome de Cushing y feocromocitoma ${ }^{3}$. Después de este caso preliminar varios grupos han descrito diferentes abordajes con técnica laparoscópica para la glándula suprarrenal, variando la posición del paciente y la técnica utilizada ${ }^{4,5}$. Gaur por ejemplo, propuso la disección y desarrollo del espacio retroperitoneal mediante el uso de un balón disector, planteando la retroperitoneoscopía como alternativa ${ }^{6}$.

En la actualidad, la literatura médica provee evidencia objetiva de que las técnicas quirúrgicas mínimamente invasivas han tenido un dramático impacto en la cirugía adrenal. Actualmente, excepto para el carcinoma suprarrenal, la cirugía laparoscópica es considerada la técnica de elección en el tratamiento de la mayoría de pacientes con entidades quirúrgicas de la glándula suprarrenal $^{1,7,8}$.

Presentamos nuestra experiencia en adrenalectomía laparoscópica, después de 10 años de adoptar la técnica laparoscópica como primera opción en la cirugía suprarrenal.

\section{MATERIAL Y MÉTODO}

Se incluyeron 200 cirugías adrenales laparoscópicas realizadas en forma consecutiva en 183 pacientes con patología quirúrgica suprarrenal entre noviembre de 1994 y noviembre de 2005. Las variables analizadas fueron: sexo, edad, diagnóstico clínico e histopatológico, tamaño de la lesión, técnica quirúrgica, tiempo operatorio, sangrado intraoperatorio, complicaciones, conversiones a cirugía abierta y tiempo de hospitalización.

Preoperatoriamente el tamaño de la glándula o del tumor fue evaluado mediante tomografía computada o resonancia magnética, aunque el tamaño definitivo reportado corresponde al diámetro mayor de la pieza quirúrgica registrado por el patólogo.

Todos los procedimientos fueron realizados por vía transperitoneal con la técnica descrita previamente por nosotros ${ }^{9}$. En el caso de las adrenalectomías del lado izquierdo se utilizaron tres trócares ubicados 2-3 cm por debajo del reborde costal: un trócar de $10 \mathrm{~mm}$ en la línea axilar anterior para la óptica, y dos trócares de trabajo, uno en la línea medio clavicular y otro en la línea axilar posterior (Fig. 1). En estos casos fue necesario movilizar el ligamento esplenocólico, el ángulo esplénico del colon, el bazo y la cola del páncreas para visualizar la glándula adrenal. Para los procedimientos del lado derecho se adicionó un cuarto trócar de $5 \mathrm{~mm}$ en epigastrio, con el objetivo de avanzar una pinza para separar el hígado (Fig. 2). En estos casos, se secciona el

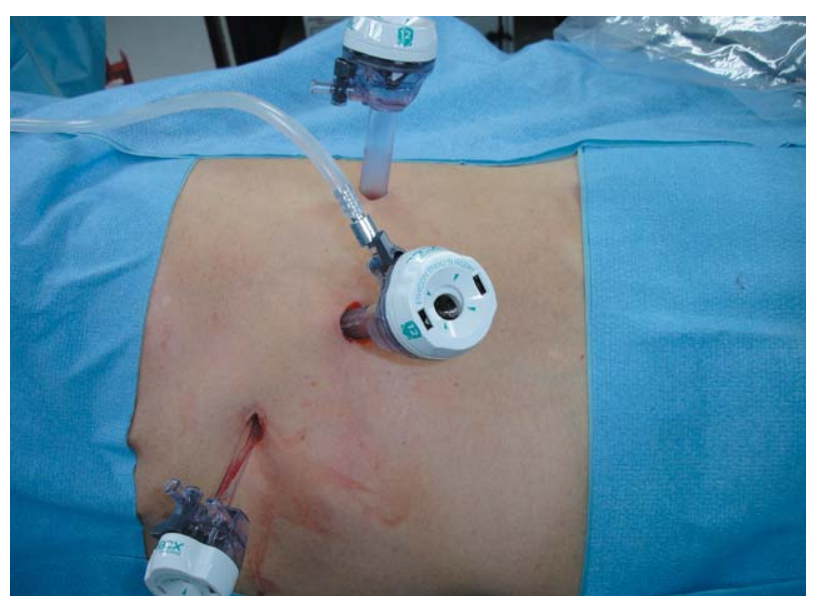

FIGURA 1. Disposición de los puertos para adrenalectomía laparoscópica izquierda. 


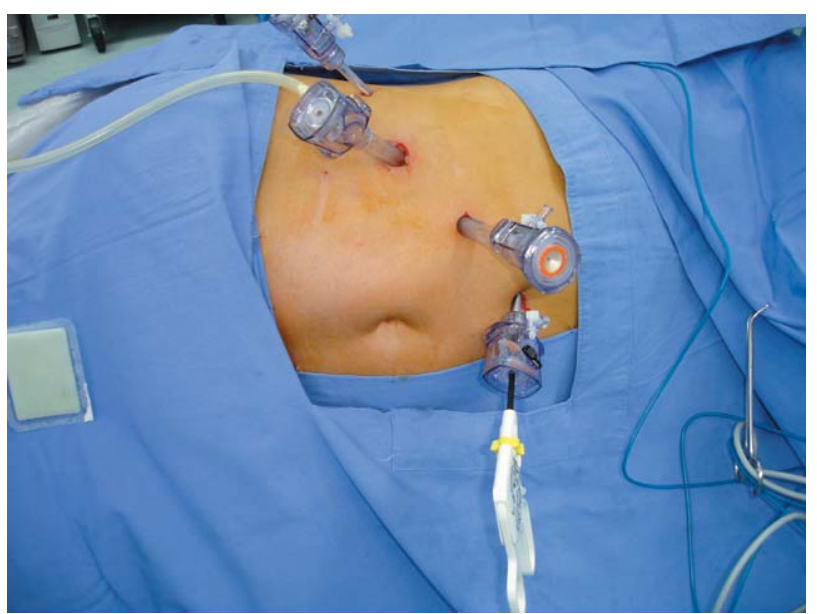

FIGURA 2. Disposición de los puertos para adrenalectomía laparoscópica derecha.

ligamento triangular hepático, lo cual permite movilizar anteriormente el hígado, se incide el peritoneo posterior transversalmente, inmediatamente caudal a lo largo de la superficie hepática, se extiende la disección por la línea de Toldt lateralmente, caudalmente sobre la superficie del polo superior del riñón y medialmente hasta la vena cava inferior. En los procedimientos derechos e izquierdos la vena suprarrenal principal es disecada, ligada y seccionada. La pieza es extraída en una bolsa de polietileno ampliando uno de los sitios de punción.

\section{RESULTADOS}

Se practicaron 200 cirugías laparoscópicas adrenales en 183 pacientes, en 17 pacientes se realizó cirugía bilateral. Fueron 67 (36,6\%) hombres y $116(63,4 \%)$ mujeres, con una edad promedio de 49,1 años (rango: 8 meses - 78 años). En 60 pacientes $(32,8 \%)$ la cirugía fue indicada por el hallazgo incidental de una masa suprarrenal en estudios imagenológicos abdominales. Los diagnósticos clínicos más frecuentes fueron hiperaldosteronismo $(17,5 \%)$, cáncer metastásico $(15,8 \%)$, feocromocitoma $(15,3 \%)$, sindrome de Cushing $(7,1 \%)$, quiste adrenal $(4,9 \%)$ y mielolipoma $(2,7 \%)$ (Tabla 1$)$.

El 58,5\% de los pacientes intervenidos tenía algún factor de comorbilidad. La hipertensión arterial fue el factor más frecuentemente asociado $(35,5 \%)$, seguido de obesidad $(13,1 \%)$, diabetes mellitus $(5,5 \%)$ y cardiopatía coronaria $(1,6 \%)$ (Tabla 2).
Tabla 1

Diagnóstico preoperatorio en 200 cirugías adrenales laparoscópicas realizadas en forma consecutiva en 183 pacientes con patología quirúrgica suprarrenal.

\begin{tabular}{lcc}
\hline Diagnóstico preoperatorio & Número & Porcentaje \\
\hline Incidentaloma & 60 & 32,8 \\
Hiperaldosteronismo & 32 & 17,5 \\
Cáncer Metastásico & 29 & 15,8 \\
Feocromocitoma & 28 & 15,3 \\
Cushing & 13 & 7,1 \\
Quiste & 9 & 4,9 \\
Mielolipoma & 5 & 2,7 \\
Tumor Virilizante & 4 & 2,2 \\
Hiperplasia Congénita & 3 & 1,6 \\
Total & 183 & 100 \\
\hline
\end{tabular}

Tabla 2

Factores de comorbilidad en 183 pacientes llevados a 200 cirugías adrenales laparoscópicas.

\begin{tabular}{lcc}
\hline Factores de Comorbilidad & Número & Porcentaje \\
\hline Hipertensión Arterial & 65 & 35,5 \\
Obesidad & 24 & 13,1 \\
Diabetes Mellitus & 10 & 5,5 \\
Cardiopatía Coronaria & 3 & 1,6 \\
Enfermedad Pulmonar Crónica & 2 & 1,1 \\
Neumonectomía & 1 & 0,5 \\
SIDA & 1 & 0,5 \\
Embarazo & 1 & 0,5 \\
\hline
\end{tabular}

Se realizaron 164 adrenalectomías totales, 29 adrenalectomías parciales y 7 marsupializaciones de quistes adrenales. Noventa y cuatro procedimientos fueron del lado derecho y 106 del lado izquierdo. El tiempo operatorio promedio fue de 82,6 minutos (rango: 25-240 minutos) y el tiempo de hospitalización fue de 2,5 días (rango: 1-10 días). El tamaño de la glándula y/o tumor suprarrenal varió entre 1 y $14 \mathrm{~cm}$ (promedio: $5,6 \mathrm{~cm}$ ).

La tasa de complicaciones fue de 6\%. Las complicaciones más frecuentes fueron por sangrado: 5 casos de sangrado intraoperatorio $(2,5 \%)$ que ameritaron transfusión sanguínea y 2 casos de hematoma retroperitoneal (1\%). Las otras complicaciones correspondieron a 2 casos de desgarro de la vena renal principal y casos únicos de fístula pancreática, lesión del diafragma, laceración del bazo y muerte. 
La fístula pancreática se presentó en una paciente operada por un adenocarcinoma renal y una masa suprarrenal izquierda metastásica de $5 \mathrm{~cm}$ de diámetro, a quien se le realizó adrenalectomía y tumorectomía renal. La fístula se manejó con drenaje percutáneo y posteriormente requirió de una papilotomía endoscópica, presentando una evolución satisfactoria.

En los casos de desgarro de la vena renal, lesión del diafragma y laceración del bazo el manejo se hizo intraoperatoriamente, con técnica laparoscópica.

La muerte intraoperatoria fue secundaria a una crisis hipertensiva en un paciente llevado a adrenalectomía derecha por un feocromocitoma de $7 \mathrm{~cm}$ de diámetro.

Del total de la serie, 6 pacientes fueron transfundidos $(3,3 \%)$. Tres pacientes recibieron una unidad de glóbulos rojos y los 3 restantes recibieron 2 unidades. Hubo un caso de conversión a cirugía abierta $(0,5 \%)$. Se trató de un paciente con síndrome de Cushing portador de una masa suprarrenal derecha de $7,5 \mathrm{~cm}$ de diámetro llevado a adrenalectomía total. La causa de la conversión fue el sangrado.

En 8 pacientes además de la cirugía adrenal, se realizó otro procedimiento quirúrgico laparoscópico: colecistectomía (2), marsupialización de quiste renal (2), nefrectomía en bloque (2), nefrectomía parcial por tumor (1) y quistectomía pancreática (1). A un paciente a quien se le realizó adrenalectomía laparoscópica derecha, se le practicó nefrolitotomía percutánea ipsilateral en el mismo acto quirúrgico.

En la Tabla 3 se muestra la distribución de pacientes de acuerdo al diagnóstico histopatológico. La entidad más frecuentemente aportada fue adenoma (29\%), seguida de hiperplasia (20\%), feocromocitoma (14\%), cáncer metastásico (12\%), mielolipoma $(7 \%)$ y carcinoma suprarrenal $(3 \%)$, entre otros.

\section{DISCUSIÓN}

A continuación, de forma resumida discutimos las entidades clínicas más frecuentes que indicaron la intervención quirúrgica.

Feocromocitoma

La mayor parte de los feocromocitomas son de presentación esporádica y no muestran un patrón
Tabla 3

Diagnóstico histopatológico en 200 cirugías adrenales laparoscópicas realizadas en forma consecutiva en 183 pacientes con patología quirúrgica suprarrenal.

\begin{tabular}{lcc}
\hline Diagnóstico Histopatológico & Número & Porcentaje \\
\hline Adenoma & 58 & 29 \\
Hiperplasia & 41 & 20,5 \\
Feocromocitoma & 29 & 14,5 \\
Cáncer Metastásico & 24 & 12 \\
Mielolipoma & 14 & 7 \\
Carcinoma Suprarrenal & 6 & 3 \\
Pseudoquiste Hemorrágico & 6 & 3 \\
Quiste Endotelial & 4 & 2 \\
Feocromocitoma Maligno & 2 & 1 \\
Otros & 16 & 8 \\
Total & 200 & 100 \\
\hline
\end{tabular}

familiar hereditario. Sin embargo, aproximadamente el $50 \%$ de los pacientes con neoplasia endocrina múltiple (MEN) 2A o 2B desarrollan un feocromocitoma. La gran mayoría de estos tumores son benignos y de localización en la glándula suprarrenal. Casi un tercio de ellos son bilaterales al momento del diagnóstico ${ }^{10}$.

$\mathrm{El}$ abordaje quirúrgico del feocromocitoma ha evolucionado acorde al entendimiento de las condiciones hemodinámicas dadas por el exceso crónico de catecolaminas y los flujos agudos de éstas en el perioperatorio. Por esta razón, para un adecuado manejo de pacientes con feocromocitoma es necesaria una óptima coordinación del clínico, anestesiólogo y cirujano ${ }^{3,11}$.

En la actualidad la cirugía laparoscópica es el tratamiento de elección y la adrenalectomía parcial es el procedimiento de preferencia en feocromocitomas bilaterales. En nuestra serie 28 pacientes fueron operados con diagnóstico clínico de feocromocitoma, 4 de ellos con lesiones bilaterales. El estudio histopatológico de las 32 piezas quirúrgicas demostró feocromocitoma en 29 de ellas y feocromocitoma maligno en 2 . De los 28 pacientes con feocromocitoma, $25(89,3 \%)$ fueron de presentación esporádica y $3(10,7 \%)$ estuvieron asociados a enfermedad de Von Hippel Lindau. Un paciente con enfermedad de Von Hippel Lindau se encontró con un quiste pancreático en el momento de la adrenalectomía, practicándosele quistectomía pancreática en el mismo 
acto operatorio. Hubo 2 complicaciones en los 29 pacientes: un caso de sangrado que requirió transfusión sanguínea en una adrenalectomía izquierda por un feocromocitoma de $10 \mathrm{~cm}$ y un caso de muerte intraoperatoria secundaria a una crisis hipertensiva en un paciente con un feocromocitoma derecho de $7 \mathrm{~cm}$ de diámetro.

En todos los casos se logró control completo de la hipertensión, con supresión de la terapia médica antihipertensiva.

\section{Cáncer Metastásico}

La adrenalectomía radical está indicada en casos de metástasis adrenales solitarias o cáncer suprarrenal primario. En pacientes con grandes metástasis adrenales solitarias o sospecha de cáncer adrenal primario, la resección quirúrgica abierta sigue siendo la técnica de elección. Sin embargo, debido a que muchas metástasis adrenales de tumores primarios extra-adrenales son pequeñas y confinadas a la glándula suprarrenal, la técnica laparoscópica ha sido considerada en estos casos específicos y varios grupos han demostrado su factibilidad $^{12}$. En nuestra experiencia, 29 pacientes fueron llevados a adrenalectomía laparoscópica por una masa considerada metastásica. El estudio histopatológico de la pieza quirúrgica demostró cáncer metastásico en 24 de ellos. Los tumores metastásicos más frecuentes fueron: pulmonar $^{7}$, melanoma $^{3}$, hipernefroma ${ }^{3}$ y linfoma ${ }^{2}$. La técnica fue reproducida en todos los pacientes, sin tener casos de conversión a cirugía abierta. Dentro de este grupo se presentó una complicación en una paciente operada por un adenocarcinoma renal y una masa suprarrenal izquierda metastásica de $5 \mathrm{~cm}$ de diámetro. Se le practicó adrenalectomía y tumorectomía renal, y en el postoperatorio se evidenció una fístula pancreática, la cual se manejó con drenaje percutáneo y posteriormente requirió de una papilotomía endoscópica.

\section{Hiperaldosteronismo Primario}

El hiperaldosteronismo primario afecta el 5$13 \%$ de los pacientes con hipertensión arterial. Los pacientes con hipertensión e hipokalemia y aquellos pacientes con hipertensión resistente al tratamiento médico deben ser tamizados para hiperaldosteronismo primario con determinación de aldosterona plasmática y actividad de renina en plasma. El hiperaldosteronismo idiopático bilateral (IHA) y el adenoma productor de aldosterona (APA) son las formas más comunes de aldosteronismo primario ${ }^{13}$. La adrenalectomía laparoscópica es una excelente opción de tratamiento para pacientes portadores de adenoma productor de aldosterona. Sin embargo, dado que la existencia de una lesión maligna en estos casos es extremadamente rara, la cirugía puede estar orientada a resecar sólo la lesión y conservar la glándula sana. Nosotros operamos 32 pacientes con hiperaldosteronismo primario y nódulo suprarrenal, 2 de ellos con nódulo bilateral. Se realizaron 35 procedimientos que correspondieron a 17 adrenalectomías totales y 18 cirugías conservadoras. En uno de los pacientes con tumor bilateral se realizó cirugía conservadora en la glándula suprarrenal izquierda, en la cual se encontró una lesión de $2,3 \mathrm{~cm}$ y adrenalectomía total derecha, donde se halló un tumor de 4,5 cm. En el otro paciente portador de masa bilateral se realizó adrenalectomía parcial bilateral. Contrario a lo aportado por otros cen$\operatorname{tros}^{14}$, en ninguno de los pacientes de este grupo hubo complicaciones operatorias, necesidad de transfusión o conversión a cirugía abierta.

En un caso de cirugía parcial hubo recurrencia del hiperaldosteronismo, siendo necesario realizar una nueva cirugía laparoscópica con extirpación completa de la glándula y curación de la hipertensión. Hubo curación completa de la hipertensión o disminución significativa de la medicación en el 85,7\% de los casos.

\section{Sindrome de Cushing}

El síndrome de Cushing describe el complejo de síntomas causados por el exceso de glucocorticoides circulantes e incluye pacientes con hipersecreción pituitaria de ACTH; pacientes con enfermedad de Cushing; aquellos con secreción ectópica de ACTH y los que se presentan con un tumor suprarrenal: adenoma o carcinoma adrenal. Los objetivos del tratamiento de pacientes con síndrome de Cushing, son disminuir la secreción diaria de cortisol a un nivel normal, erradicar cualquier tumor sin producir una deficiencia endocrina permanente y evitar la dependencia continua de medicamentos ${ }^{15}$. En el caso del síndrome de Cushing ACTH-dependiente el tratamiento quirúrgico está dirigido al tumor hipofi- 
siario o al tumor primario fuente de hormona adrenocorticotrópica ectópica, quedando indicada la adrenalectomía bilateral para aquellos casos en que el tumor fuente de hipersecreción de ACTH no puede ser identificado o removido, o cuando hay un hipercortisolismo persistente o recurrente luego de hipofisectomía ${ }^{16}$. Para los pacientes con Cushing adrenal primario, la adrenalectomía bilateral está indicada en casos de hiperplasia bilateral, mientras que la adrenalectomía unilateral es la elección en presencia de un adenoma suprarrenal o en los casos infrecuentes de carcinoma adrenal primario. En nuestra serie 13 pacientes fueron llevados a adrenalectomía laparoscópica por síndrome de Cushing. En 4 de ellos se realizó adrenalectomía bilateral indicada por Cushing adrenal primario secundario a hiperplasia bilateral en 3 pacientes y falla de cirugía hipofisiaria en 1. En 9 pacientes se practicó adrenalectomía unilateral por la presencia de un nódulo suprarrenal, todos con excelente respuesta clínica.

Dentro del grupo de pacientes operados por síndrome de Cushing, hubo un caso de sangrado intraoperatorio que ameritó conversión a cirugía abierta. Se presentó durante una adrenalectomía derecha en una mujer portadora de un adenoma de $7,5 \mathrm{~cm}$ de diámetro.

\section{Quiste Suprarrenal}

La mayoría de los quistes suprarrenales son asintomáticos y no funcionantes. Sin embargo, cuando su tamaño es considerable, pueden causar efecto de masa, dolor, sangrado o hipertensión, aunque también pueden dar la apariencia de un tumor suprarrenal en los estudios imagenológi$\cos ^{17}$. Los quistes suprarrenales pequeños, asintomáticos o no funcionantes son manejados conservadoramente. Cuando un quiste adrenal supera los $6 \mathrm{~cm}$ de diámetro, si es funcionante o sintomático, y/o en aquellos casos en los cuales hay sospecha de malignidad por su apariencia imagenológica, la exploración quirúrgica está indicada ${ }^{18}$. La técnica laparoscópica también ha sido utilizada en el caso de los quistes adrenales y nosotros recientemente publicamos nuestra experiencia ${ }^{19}$. En la serie actual, 9 pacientes fueron intervenidos por quiste suprarrenal: en 7 se realizó decorticación y marsupialización del quiste, en 1 adrenalectomía parcial y en 1 adrenalectomía total. En la totalidad de este grupo de pacientes se obtuvo resolución de los sintomas, y a la fecha, con una media de seguimiento de 26 meses, no se tiene evidencia radiográfica de recurrencia.

\section{Mielolipoma}

Muchos de los mielolipomas suprarrenales son pequeños, asintomáticos y son descubiertos incidentalmente durante estudios radiológicos. Típicamente estos tumores son menores de $5 \mathrm{~cm}$, sin embargo en ocasiones se desarrollan síntomas clínicos, comúnmente dolor abdominal cuando estas lesiones crecen o sangran ${ }^{20}$. La hemorragia retroperitoneal también es una complicación que se presenta con alguna frecuencia ${ }^{21}$. Se destaca el significativo número de pacientes con mielolipoma suprarrenal en nuestra serie: 14 en total, siendo dentro de nuestro conocimiento, la serie de adrenalectomía laparoscópica por mielolipoma más grande encontrada en la literatura. Estos tumores se caracterizan por la presencia de una pseudocápsula que facilita la disección de la masa, así como el control vascular, haciendo que la cirugía laparoscópica para estos tumores sea técnicamente menos compleja. En el grupo de pacientes operados por mielolipoma el tamaño promedio del tumor fue 9,5 cm (rango: 5-14 cm). No hubo complicaciones operatorias, necesidad de transfusión o conversión a cirugía abierta en ninguno de los 15 pacientes operados por mielolipoma.

Todos los pacientes que requirieron transfusión sanguínea $(3,3 \%)$, quienes presentaron complicaciones intraoperatorias $(6 \%)$ y el que necesitó conversión a cirugía abierta $(0,5 \%)$, correspondieron a la primera parte de la serie: casos 1 al 46 de un total de 200 procedimientos. El sexo, la edad, la indicación quirúrgica o el lado intervenido no influyeron en los resultados o en la tasa de complicaciones. El único factor que fue constante en los pacientes que cursaron con complicaciones o que requirieron transfusión fue el tamaño de la lesión: $\geq 5 \mathrm{~cm}$ de diámetro mayor.

Existe controversia sobre el uso de la técnica laparoscópica para abordar masas suprarrenales mayores de $5 \mathrm{~cm}$ de diámetro, y aunque el tamaño de la masa per se no representa una contraindicación absoluta para utilizar la vía laparoscópica, varios grupos sugieren que esta técnica quirúrgica se limite a glándulas menores de 5-6 $\mathrm{cm}^{8,22}$. 
Sin embargo, hay casos recientes en la literatura que demuestran la factibilidad y reproducibilidad de la técnica laparoscópica en pacientes con grandes masas suprarrenales ${ }^{23-26}$. En nuestra experiencia, 92 pacientes $(50,2 \%)$ fueron operados por masa $\geq 5 \mathrm{~cm}$. En este grupo de pacientes, aunque es necesaria una mayor disección y movilización quirúrgica, la técnica fue reproducida satisfactoriamente, teniendo sólo un caso de conversión a cirugía abierta. En la medida que se ganó experiencia con la técnica, se logró minimizar el sangrado y las complicaciones, consiguiendo extender los beneficios de un procedimiento mínimamente invasivo en el abordaje de grandes masas suprarrenales. En nuestra opinión, el tamaño de la masa suprarrenal no debe ser el factor primario en determinar la posibilidad de realizar una adrenalectomía con técnica laparoscópica.

Aunque existen indicaciones para adrenalectomía laparoscópica como son el aldosteronoma, feocromocitoma, enfermedad de Cushing, y entidades raras como quistes y mielolipomas, generalmente las indicaciones y contraindicaciones de la adrenalectomía laparoscópica están dadas principalmente por la experiencia del cirujano laparoscopista. Las masas de un tamaño mayor a $5 \mathrm{~cm}$ tienen más tendencia al sangrado y complicaciones mayores, sin embargo la adrenalectomía laparoscópica es una técnica factible y efectiva para el abordaje quirúrgico de grandes masas adrenales cuando el equipo quirúrgico tiene amplia experiencia en cirugía laparoscópica avanzada.

Consideramos que este significativo número de procedimientos, 200 en total, así como la variedad y complejidad de las patologias ha permitido hacer una curva de perfeccionamiento de la técnica, hasta llegar al punto actual en que el procedimiento es de mínima morbilidad, con tiempos quirúrgicos satisfactorios y una corta estancia hospitalaria postoperatoria.

\section{REFERENCIAS}

1. Gill IS. The case for laparoscopic adrenalectomy. J Urol. 2001;166:429 436.

2. Gagner M, Lacroix A, Bolte E. Laparoscopic adrenalectomy in Cushing's syndrome and pheochromocytoma. N Engl J Med. 1992;327:10331036.

3. Quan-Yang D. Evolving surgical management for patients with pheochromocytoma. J Clin Endocrinol Metab. 2001;86:1477-1479.

4. Guazzoni G, Cestari A, Montorsi F, Bellinzoni P, Centemero A, Naspro $\mathrm{R}$, et al. Laparoscopic treatment of adrenal diseases: 10 years on. BJU International 2004;93:221-227.
5. Salomon L, Soule M, Mouly P, Saint F, Cicco A, Abbou CC, et al. Experience with retroperitoneal laparoscopic adrenalectomy in 115 procedures. J Urol. 2001;166:38-41.

6. Gaur DD. Laparoscopic operative retroperitoneoscopy. Use of a new device. J Urol. 1992;148:1137-1142.

7. Gagner M, Pomp A, Heniford B, Pharand D, Lacroix A. Laparoscopic adrenalectomy: lessons learned from 100 consecutive procedures. Ann Surg. 1997;226:238-247.

8. Winfield H, Hamilton B, Bravo E, Novick A. Laparoscopic adrenalectomy: the preferred choice? A comparison to open adrenalectomy. J Urol.1998; 160:325-329.

9. Castillo O, Rossi R, Amat J, Van Cauverlaert R, Poblete P, Rodriguez F, et al. Adrenalectomía Laparoscópica: Resultados de una experiencia chilena inicial. Rev Méd Chil. 1999;127:304-308.

10. Pacak K, Ilias I, Adams KT, Eisenhofer G. Biochemical diagnosis, localization and management of pheochromocytoma: focus on multiple endocrine neoplasia type 2 in relation to other hereditary syndromes and sporadic forms of the tumour. J Int Med. 2005;257:60-68.

11. Bravo EL, Tagle R. Pheochromocytoma: State of the art and future prospects. Endocr Rev. 2003;24:539-553.

12. Moinzadeh A, Gill I. Laparoscopic radical adrenalectomy for malignancy in 31 patients. $J$ Urol. 2005;173:519-525.

13. Young WF Jr. Minireview: Primary aldosteronism-Changing concepts in diagnosis and treatment. Endocrinology 2003;144:2208-2213.

14. Meria P, Kempf BF, Hermieu JF, Plouin PF, Duclos JM. Laparoscopic management of primary Hyperaldosteronism: clinical experience with 212 cases. J Urol. 2003;169:32-35.

15. Orth DN. Cushing's syndrome. N Engl J Med. 1995;332:791-797.

16. Vella A, Thompson GB, Grant CS, Vanheerden JA, Farley DR, Young WF Jr. Laparoscopic adrenalectomy for adrenocorticotropin-dependent Cushing’s syndrome. J Clin Endocrinol Metab. 2001;86:1596-1599.

17. Karayiannakis AJ, Plychronidis A, Simopoulos C. Giant adrenal pseudocyst presenting with gastric outlet obstruction and hypertension. Urology 2002;59:946-947.

18. Wang LJ, Wong YC, Chen CJ, Chu SH. Imaging spectrum of adrenal pseudocyst on CT. Eur Radiol. 2003;13:531-533.

19. Castillo OA, Litvak JP, Kerkebe M, Ureña R. Laparoscopic management of symptomatic and large adrenal cysts. J Urol. 2005;173:915-917.

20. Meaglia J, Schmidt J. Natural history of an adrenal myelolipoma. J Urol. 1992;147:1089-1090.

21. El-Mekresh M, Abdel-Gawad M, El-Diasty T, El-Baz M, Ghoneim M. Clinical, radiological and histological features of adrenal myelolipoma: review and experience with a further eight cases. Br J Urol. 1996;78: 345-350.

22. Hazzan D, Shiloni E, Goliljanin D. Laparoscopic vs. Open Adrenalectomy for benign adrenal neoplasm: A comparative study. Surg Endosc. 2001;15:1356-1358.

23. Hobart M, Gill I, Schweizer D, Sung G, Bravo E. Laparoscopic adrenalectomy for large-volume $(>/=5 \mathrm{~cm})$ adrenal masses. J Endourol 2000;14:149-154.

24. Mcgillivray DC, Whalen Gf, Malchoff CD, Oppenheim DS, Shichman SJ. Laparoscopic resection of large adrenal tumors. Ann Surg Oncol. 2002;9:480-485.

25. Castillo O, Cortes O, Pinto I, Portalier P, Diaz M, Castillo D, Cardia F.: Laparoscopic adrenalectomy for large adrenal masses: experience with 51 procedures. J Endourology 2003; 17 (Supplement 1): Abstract MP10.10, page A70.

26. Novitsky Y, Czerniach D, Kercher K W, Perugini R, Kelly J, Litwin D. Feasibility of laparoscopic adrenalectomy for large adrenal masses. Surg Laparosc Endosc Percutan Tech. 2003;13:106-110.

Dr. O. Castillo

Unidad de Endourología y Laparoscopia Urológica.

Clínica Santa María

Avda. Santa María 0500, Providencia Santiago, Chile

E-mail: octaviocastillo@vtr.net

(Trabajo recibido el 25 de enero de 2006) 